\title{
Piezoelectric power extraction from bending waves: Electroelastic modeling, experimental validation, and performance enhancement
}

\author{
S. Tol, F.L. Degertekin, and A. Erturk ${ }^{*}$ \\ G. W. Woodruff School of Mechanical Engineering, Georgia Institute of Technology, Atlanta,
} GA 30332, USA

\begin{abstract}
Existing research in vibration-based energy harvesting has focused mostly on the harvesting of deterministic or stochastic vibrational energy available at a fixed position in space. Such an approach is convenient for designing and employing linear and nonlinear vibration-based energy harvesters, such as base-excited cantilevers with piezoelectric laminates. This work presents a mathematical framework for the harvesting of one-dimensional bending waves propagating in infinite and semi-infinite beams as an alternative. For this purpose, the fully coupled electroelastic problem with piezoelectric patches bonded to a long slender beam is solved and conversion of incident wave energy into usable electricity while minimizing the traveling waves reflected and transmitted from the harvester domain is analyzed. The analysis shows that the efficiency of power transfer from elastic waves can be significantly improved beyond the typical wavelength matching in terms of both efficiency and bandwidth by inductive-resistive loading. It is also shown that enhancements to efficiency can be obtained by localized obstacles in mechanical domain, and fully anechoic boundary conditions can be obtained on finite beams by resistive-inductive impedance matching. These enhancement methods are most effective and

${ }^{*}$ Corresponding author. E-mail address: alper.erturk@me.gatech.edu; Tel.: +1 4043851394

Address: George W. Woodruff School of Mechanical Engineering, Georgia Institute of Technology, Atlanta, GA 30332.
\end{abstract}


practical when piezoelectric patch lengths and obstacle to patch distances are $\sim>\lambda / 4$, where evanescent fields become insignificant, while the model can readily accommodate the presence of evanescent waves for arbitrary patch lengths. The validity and application of the proposed methods are demonstrated with experimental case studies using a long slender beam.

Keywords: Piezoelectricity, energy harvesting, wave propagation, power flow, impedance matching.

\section{Introduction}

Over the last decade, several research groups have worked on energy harvesting in order to power small electronic components by scavenging ambient energy available in their environment. The ultimate goal of these efforts is to create self-powered electronic devices for various wireless applications ranging from structural health monitoring sensors to medical implants [1-4]. Ambient energy exists in various forms, such as waste heat, solar, vibration, and flow energy. While each of these sources of energy can be used to power remote sensors, the harvesting of vibrational energy has been mostly studied as a viable alternative source [5-10]. Among different transduction mechanisms that can be used for vibration-to-electric energy conversion, piezoelectric transduction has become the most popular method due to the ease of application, the high power density, and the relative maturity of the manufacturing methods of piezoelectric materials at different scales [11]. Most of the piezoelectric energy harvesters are in the form of unimorph or bimorph cantilevered beams. An alternating voltage output is obtained by applying harmonic base motion to the structure, and maximum power is generated typically at the fundamental resonant frequency of the composite structure [12]. Although it is a common practice to characterize the resonance behavior of a piezoelectric energy harvester with harmonic 
excitation, different models may be required for other loading conditions, especially in the absence of standing waves. For instance, scavenging energy from vibrations due to fluidstructure interaction requires a more complex analysis because of the coupling of piezoelastic structure with the surrounding airstream [3]. In literature, flow energy harvesting through aeroelastic [13-20] and hydroelastic [21-23] vibrations has been studied. Yet, researchers have given little effort to exploiting the energy of traveling waves in fluids and structures. Few research groups have studied this area with a focus on polarization-patterned piezoelectric solids [24], quarter-wavelength resonators [25], Helmholtz resonators [26, 27], or phononic crystals [28-31]. In addition, Yang et al. [32] combined the sonic crystal concept with the Helmholtz resonators to improve acoustic energy harvesting. Furthermore, acoustic metamaterials can be used to enhance energy harvesting by changing wave propagation. For instance, Carrara et al. $[33,34]$ implemented metamaterial-inspired structures in order to guide, localize, and focus elastoacoustic waves for more efficient piezoelectric energy harvesting. Since wave equation is the common theoretical basis for metamaterial-based elastoacoustic and electromagnetic energy harvesting, the combination of structural configurations with metamaterials and wave propagation is essential for performance enhancement [35].

We also note that various researchers combined the wave propagation theory with the piezoelectric sensing/actuating mechanisms to study passive and active structural health monitoring (SHM) systems [36-39]. For instance, the electromechanical impedance technique for SHM utilizes travelling waves and the electrical impedance of a surface-bonded PZT transducer to obtain the change in the electromechanical impedance signature of the structure and detects the structural damage by monitoring this change [40-43]. In various SHM applications, researchers utilized ultrasonic waves such as Rayleigh surface waves and Lamb 
modes $[36,44,45]$. In these studies, the focus has been either only on the forward problem to excite these waves more efficiently, or to sense with small size piezo patches where the important factor is signal to noise, not efficiency of energy transfer. In those cases, for example, the effect of piezo patch electrical impedance and load termination on the propagating wave amplitudes through backward coupling are not considered.

Our study fully combines the bending waves and the piezoelectric theory in the wave energy harvester to investigate the energy transfer from propagating wave energy in the structures to resistive and reactive electrical loads. As a specific and tractable example, piezoelectric patches bonded to a slender thin beam are considered. Incident wave energy is transformed into usable electricity while minimizing the traveling waves reflected and transmitted from the harvester domain. Electroelastic models are developed for the harvester bonded to infinite and semi-infinite beams by implementing the wave equation solution in the compatibility and equilibrium conditions at the harvester boundaries which are then solved simultaneously with the coupled electrical equation yielding the amplitudes of the traveling waves and the voltage response of the harvester. Hence, both electrical to mechanical and mechanical to electrical coupling problems are simultaneously solved. This allows one to obtain harvested power and the harvester efficiency for different electrical loading conditions and performance enhancement by wavelength matching, resistive-inductive circuits, and spatially localized obstacles. Additionally, an energy-harvesting end condition in a semi-infinite beam is introduced to minimize the reflection in the sense of creating ideally an anechoic boundary condition. The validity and application of the proposed model and the performance enhancement methods are demonstrated with several experimental studies by using a long slender beam. 


\section{Electroelastic modeling}

When a wave, propagating along a waveguide such as a beam, encounters discontinuity, it is reflected and transmitted across that discontinuity [46]. As shown in Fig. 1, piezoelectric patches, symmetrically bonded to the top and bottom of a thin beam, are the sources of the discontinuity. The reflected and transmitted wave properties can be obtained from wave elastodynamics in the beam, as explained in the first section. In the second section, piezoelectric

energy harvesting is combined with the wave propagation theory, and the power flow to the electrical load through the piezoelectric patches is extracted. In the third section, a lumped mass is introduced to the system in order to increase the energy harvested in the piezoelectric patches. Finally, in the last section, the energy harvester is implemented at the end of the structure with a resistive-inductive circuit, resulting in a multifunctional energy harvester which ideally realizes an anechoic boundary condition while converting all incident elastic energy to electrical domain.

\subsection{Bending waves in an infinite Euler-Bernoulli beam}

The unforced bending wave equation for one-dimensional thin beam with constant crosssectional area can be written as

$$
Y I \frac{\partial^{4} w(x, t)}{\partial x^{4}}+m \frac{\partial^{2} w(x, t)}{\partial t^{2}}=0
$$

where $Y I$ is the flexural rigidity of the beam, $m$ is the mass per unit length, and $w(x, t)$ is the transverse displacement. This equation is based on the classical (Euler-Bernoulli) beam theory, which postulates that plane sections remain plane (by neglecting shear distortion) and also excludes the rotary inertia effects. The complete complex solution for the homogenous problem can be obtained as 


$$
w(x, t)=\left(\tilde{A} e^{-j k_{b} x}+\tilde{B} e^{j k_{b} x}+\tilde{C} e^{-k_{b} x}+\tilde{D} e^{k_{b} x}\right) e^{j \omega t}
$$

where $k_{b}=\left(\omega^{2} m / Y I\right)^{1 / 4}$ is the bending wave number, $\omega$ is the frequency, and $j$ is the unit imaginary number. The first two terms on the right-hand side of Eq. (2) represent waves propagating in the positive and negative $x$ directions at a phase velocity of $c_{b}=\omega / k_{b}$. The last two terms represent evanescent waves with exponentially decaying amplitudes with distance. Those waves do not individually transport energy, but can be effective and should be considered when the distance between discontinuities significantly smaller than the wavelength as this impacts the minimum patch size for practical optimization of power transfer.

In the energy harvesting problem, two identical piezoelectric patches of length $L$ and thickness $h_{p}$ are bonded (with a negligible bonding layer) to top and bottom surfaces of the thin beam for energy harvesting, forming a symmetric structure as shown in Fig. 1. As a result, the incident wave $w_{i}^{+}$(propagating from the left end of the beam) is scattered, generating various transmitted and reflected waves in three regions.
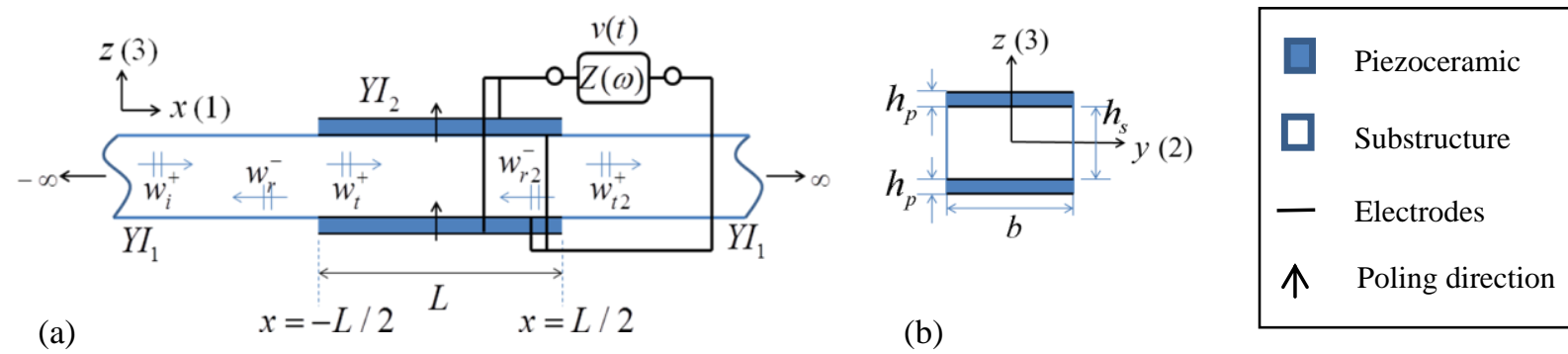

Fig. 1. Incident, reflected, and transmitted waves at discontinuities: (a) Parallel connection and (b) composite cross-section in the harvester region.

Based on Fig. 1, the incident, reflected and transmitted waves can be expressed as 


$$
\begin{gathered}
w_{i}^{+}(x, t)=\tilde{A} \mathrm{e}^{-j k_{b 1} x} e^{j \omega t} \\
w_{r}^{-}(x, t)=\left(\tilde{B}_{1} \mathrm{e}^{+j k_{b 1} x}+\tilde{B}_{2} \mathrm{e}^{+k_{b 1} x}\right) e^{j \omega t} \\
w_{t}^{+}(x, t)=\left(\tilde{C}_{1} \mathrm{e}^{-j k_{b 2} x}+\tilde{C}_{2} \mathrm{e}^{-k_{b 2} x}\right) e^{j \omega t} \\
w_{r 2}^{-}(x, t)=\left(\tilde{D}_{1} \mathrm{e}^{+j k_{b 2} x}+\tilde{D}_{2} \mathrm{e}^{+k_{b 2} x}\right) e^{j \omega t} \\
w_{t 2}^{+}(x, t)=\left(\tilde{E}_{1} \mathrm{e}^{-j k_{b 1} x}+\tilde{E}_{2} \mathrm{e}^{-k_{b 1} x}\right) e^{j \omega t}
\end{gathered}
$$

where $\tilde{B}_{1}, \tilde{C}_{1}, \tilde{D}_{1}, \tilde{E}_{1}$ are the complex amplitudes of the propagating wave components while $\tilde{B}_{2}, \tilde{C}_{2}, \tilde{D}_{2}, \tilde{E}_{2}$ are the complex amplitudes of the evanescent counterparts, and the + and superscripts indicate propagation in the $+x$ and $-x$ directions, respectively.

The shear force $Q$ and bending moment $M$ are

$$
Q(x, t)=-Y I \frac{\partial^{3} w(x, t)}{\partial x^{3}} \quad M(x, t)=-Y I \frac{\partial^{2} w(x, t)}{\partial x^{2}}
$$

The complex wave amplitudes are obtained by imposing the linear/angular displacement compatibility and force/moment equilibrium conditions at $x=-L / 2$ and $x=L / 2$ and solving the resulting equations simultaneously [47]. With the known complex wave amplitudes, the time averaged power flow in the beam at any position can be calculated by using

$$
P_{\text {avg }}=\frac{1}{2} \operatorname{Re}\left(-\dot{w}^{*} Q-\dot{\theta}^{*} M\right)
$$

where $\dot{w}^{*}$ is the complex conjugate of the velocity phasor and $\dot{\theta}^{*}$ is the complex conjugate of the angular velocity phasor [48]. When the evanescent fields are ignored, this expression converges to simpler expressions obtained for bending waves in the literature [49].

Also note that, in order to account for the mechanical losses in the system, structural damping can be introduced in the model by attributing a complex elastic modulus to the 
material, $Y_{s}^{*}=Y_{s}(1+j \eta)$, where $\eta$ is the structural damping factor, which is generally much smaller than unity [47]. When this complex elastic modulus is substituted in Eq. (1), the solution of the homogenous wave equation gives a complex bending wave number which can be approximated by $k_{b}^{*}=k_{b}(1-j \eta / 4)$.

\subsection{Piezoelectric energy harvesting from one-dimensional bending waves}

Energy harvesting is accomplished when the piezoelectric patches with electrodes are connected to a finite, non-zero electrical load, $Z_{l}(\omega)$ (which may have purely resistive or resistive-reactive components $[50,51])$. In this study, the harvesting model applies to both parallel and series connections of piezoceramic layers for the symmetric bimorph configuration. In Fig. 1, the parallel connection is shown (see the Appendix for the equivalent representations of the series connection of piezoceramic layers). The $x, y$, and $z$ directions are coincident with the 1, 2, and 3 directions (conventionally the poling direction is the 3 -direction). The bending moment can be written for the piezoelectrically coupled configuration as [3]

$$
M(x, t)=b\left(\int_{-h_{p}-h_{s} / 2}^{-h_{s} / 2} T_{1}^{p} z d z+\int_{-h_{s} / 2}^{h_{s} / 2} T_{1}^{s} z d z+\int_{h_{s} / 2}^{h_{p}+h_{s} / 2} T_{1}^{p} z d z\right)
$$

where $b$ is the width, and $h_{p}$ and $h_{s}$ are the thicknesses of the piezoceramic and substructure layer, respectively. Hereafter, the subscripts and superscripts $p$ stands for the piezoceramic layers and $s$ stands for the substructure layer. Furthermore, $T_{1}^{p}$ and $T_{1}^{s}$ are the stress components (in $x$-direction) in the piezoceramic and substructure layers, respectively. These stress components can be written as follows:

$$
T_{1}^{p}=\bar{c}_{11}^{E} S_{1}^{p}-\bar{e}_{31} E_{3}, \quad T_{1}^{s}=Y_{s} S_{1}^{s}
$$


where $\bar{c}_{11}^{E}$ is the elastic modulus of piezoceramic at constant electric field, $\bar{e}_{31}$ is the effective piezoelectric stress constant, and $Y_{s}$ is the elastic modulus of the substructure, $S_{1}{ }^{p}$ and $S_{1}^{s}$ are the axial strain components $\left(S_{1}(x, z, t)=-z\left(\partial^{2} w(x, t) / \partial x^{2}\right)\right)$ and $E_{3}$ is the electric field component in $z$-direction. The piezoceramic layers are assumed to be identical; hence, the voltage across the electrodes of each piezoceramic layer is $v(t)$ in the parallel connection case. Since $\bar{e}_{31}$ has the same sign in both layers, the instantaneous electric fields are in opposite directions for the parallel connection (i.e., $E_{3}(t)=-v(t) / h_{p}$ in the top layer and $E_{3}(t)=v(t) / h_{p}$ in the bottom layer).

Substituting Eqs. (7) into Eq. (6), the bending moment for the composite cross-section is obtained as

$$
M(x, t)=-Y I_{2} \frac{\partial^{2} w(x, t)}{\partial x^{2}}+\chi v(t)
$$

where $Y I$ is the flexural rigidity (bending stiffness) term of the composite cross-section under the short-circuit (or constant electric field) condition [3] and $\chi$ is the backward coupling term (that accounts for the feedback effect of electrical domain on the elastoacoustic domain), which can be defined as

$$
\chi=\frac{b \bar{e}_{31}}{h_{p}}\left[\left(h_{p}+\frac{h_{s}}{2}\right)^{2}-\left(\frac{h_{s}}{2}\right)^{2}\right]=2 b \bar{e}_{31} h_{p c}
$$

Here, $h_{p c}$ is the distance between the neutral axis and the center of each piezoceramic layer in the harvester region $\left(h_{p c}=\left(h_{p}+h_{s}\right) / 2\right)$.

For the composite cross-section, the moment equilibrium equations at the boundaries of the patch are modified according to the coupled mechanical equation, Eq. (8). 


$$
\begin{gathered}
-\left.Y I_{1}\left(\frac{\partial^{2} w_{i}^{+}}{\partial x^{2}}+\frac{\partial^{2} w_{r}^{-}}{\partial x^{2}}\right)\right|_{x=-L / 2}=-\left.Y I_{2}\left(\frac{\partial^{2} w_{t}^{+}}{\partial x^{2}}+\frac{\partial^{2} w_{r 2}^{-}}{\partial x^{2}}\right)\right|_{x=-L / 2}+\chi v(t) \\
-\left.Y I_{2}\left(\frac{\partial^{2} w_{t}^{+}}{\partial x^{2}}+\frac{\partial^{2} w_{r 2}^{-}}{\partial x^{2}}\right)\right|_{x=L / 2}+\chi v(t)=-\left.Y I_{1} \frac{\partial^{2} w_{t 2}^{+}}{\partial x^{2}}\right|_{x=L / 2}
\end{gathered}
$$

When the piezoelectric patches are connected to a load impedance $Z_{l}(\omega)$, which can consist of resistance and inductance, the system becomes electromechanically coupled, resulting in a ninth unknown term, $v(t)=\tilde{V} e^{j \omega t}$, voltage response of this coupled system. The coupled electrical circuit equation (the ninth governing equation) for this system can be written as [3]

$$
j \omega C_{p}^{e q} \tilde{V}+\frac{\tilde{V}}{Z_{l}(\omega)}-\tilde{I}_{p}=0
$$

where $C_{p}^{e q}$ is the equivalent capacitance of the piezoelectric patches in parallel connection due to two identical capacitors $\left(\bar{\varepsilon}_{33}^{S} b L / h_{p}\right)$ connected in parallel.

$$
C_{p}^{e q}=2 \bar{\varepsilon}_{33}^{S} b L / h_{p}
$$

Here, $\bar{\varepsilon}_{33}^{S}$ is the permittivity component at constant strain with the plane-stress assumption for the patch. In order to account for dielectric loss tangent $\tan \delta$, complex permittivity can be used conveniently to give $\bar{\varepsilon}_{33}^{S}=\bar{\varepsilon}_{33}^{S}(1-j \tan \delta)$, yielding a complex capacitance expression. The dependent current source can be expressed as

$$
i_{p}(t)=\tilde{I}_{p} e^{j \omega t}=-2 \bar{e}_{31} h_{p c} b \int_{-L / 2}^{L / 2} \frac{\partial^{3} w_{2}(x, t)}{\partial x^{2} \partial t} d x
$$

where $w_{2}(x, t)=w_{t}^{+}(x, t)+w_{r 2}^{-}(x, t)$ is the total wave in the composite section. The amplitude of the current in Eq. (12) can be obtained as $\tilde{I}_{p}=-j \omega \kappa$ with $\kappa$ being the forward coupling term 
(that accounts for the excitation of electrical circuit by elastoacoustic waves), which can be extracted from Eq. (14) as

$$
\kappa=4 k_{b 2} \bar{e}_{31} h_{p c} b\left(-\left(\tilde{C}_{1}+\tilde{D}_{1}\right) \sin \left(k_{b 2} \frac{L}{2}\right)+\left(\tilde{C}_{2}+\tilde{D}_{2}\right) \sinh \left(k_{b 2} \frac{L}{2}\right)\right)
$$

Therefore this set of equations fully model the forward and backward coupling in this wave based electromechanical system. Note that ignoring backward coupling and evanescent waves, Eq. (15) suggests that energy conversion will be effective at odd multiples of $\lambda / 2$. This is an expected result as shown by others for $A_{0}$ mode Lamb waves, but as will be shown later both the backward coupling and evanescent waves are significant in determining optimal parameters for energy conversion [45].

After the complex wave amplitudes and voltage response are obtained by solving the coupled electroelastic system, the time averaged electrical power flow into the electrical load can be calculated as

$$
P_{e_{\text {avg }}}=\frac{|\tilde{V}|^{2}}{2 R_{l}}
$$

This expression along with Eq. (5) can be used to verify the power balance in the system, determine the power flow into the harvester, and hence evaluate the efficiency of the energy harvester from the known input mechanical power to the system.

\subsection{Harvester with a lumped obstacle}

Piezoelectric energy harvesting from propagating bending waves on beams with an integrated symmetrical obstacle can be modeled using the schematic shown in Fig. 2. The goal of this approach is to recover and harvest part of the transmitted wave energy, which can also be interpreted as creating a spatially localized standing wave pattern. In the following, it is assumed 
that the lateral extent of the obstacle is much smaller than the wavelength, and thus a lumped modeling approach can be used.

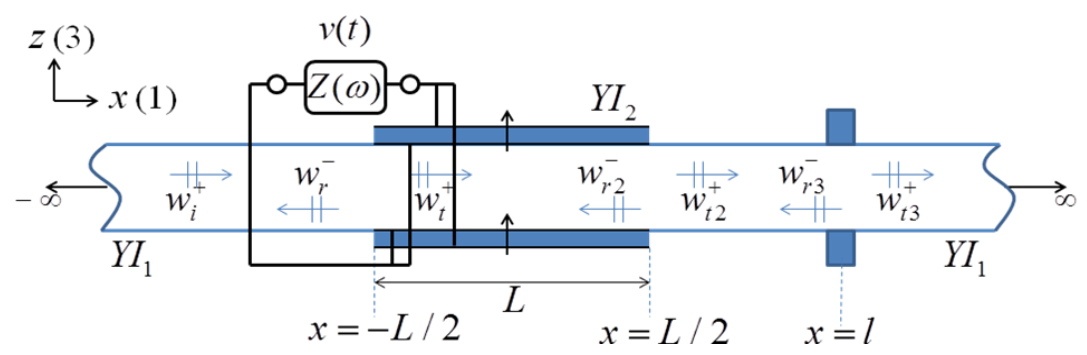

Fig. 2. Piezoelectric energy harvesting from one-dimensional bending waves in the presence of a lumped obstacle.

When the transmitted wave $\left(w_{t 2}^{+}\right)$from the harvester is incident upon the obstacle, it gives rise to reflected $\left(w_{r 3}^{-}(x, t)=\left(\tilde{F}_{1} \mathrm{e}^{+j k_{b 1} x}+\tilde{F}_{2} \mathrm{e}^{+k_{b 1} x}\right) e^{j \omega t}\right)$ and transmitted waves $\left(w_{t 3}^{+}(x, t)=\left(\tilde{G}_{1} \mathrm{e}^{-j k_{b 1} x}+\tilde{G}_{2} \mathrm{e}^{-k_{b 1} x}\right) e^{j \omega t}\right)$ for this additional discontinuity.

Wave amplitudes and the voltage response are obtained by imposing the linear/angular displacement compatibility and force/moment equilibrium conditions at $x=-L / 2, x=L / 2$ and $x=l$ and solving the resulting equations with the coupled electrical circuit equation simultaneously. The same equations at $x=-L / 2$ are still valid, while at $x=L / 2$ reflected wave from the obstacle $\left(w_{r 3}^{-}\right)$is added to the compatibility and equilibrium equations.

The linear/angular displacement compatibility and force/moment equilibrium conditions at $x=l$ can be written as

$$
w_{t 2}^{+}(l)+w_{r 3}^{-}(l)=w_{t 3}^{+}(l)
$$




$$
\begin{gathered}
\left.\left(\frac{\partial w_{t 2}^{+}}{\partial x}+\frac{\partial w_{r 3}^{-}}{\partial x}\right)\right|_{x=l}=\left.\frac{\partial w_{t 3}^{+}}{\partial x}\right|_{x=l} \\
\left.Y I_{1}\left(\frac{\partial^{3} w_{t 2}^{+}}{\partial x^{3}}+\frac{\partial^{3} w_{r 3}^{-}}{\partial x^{3}}\right)\right|_{x=l}=\left.Y I_{1} \frac{\partial^{3} w_{t 3}^{+}}{\partial x^{3}}\right|_{x=l}+\left.m_{o} \frac{\partial^{2} w_{t 3}^{+}}{\partial t^{2}}\right|_{x=l} \\
-\left.Y I_{1}\left(\frac{\partial^{2} w_{t 2}^{+}}{\partial x^{2}}+\frac{\partial^{2} w_{r 3}^{-}}{\partial x^{2}}\right)\right|_{x=l}=-\left.Y I_{1} \frac{\partial^{2} w_{t 3}^{+}}{\partial x^{2}}\right|_{x=l}+\left.I_{o} \frac{\partial^{3} w_{t 3}^{+}}{\partial x \partial t^{2}}\right|_{x=l}
\end{gathered}
$$

where $m_{o}$ is the total mass, and $I_{o}$ is the total mass moment of inertia of the lumped obstacle.

\subsection{Harvester at the boundary of a semi-infinite beam: Electromechanical anechoic}

\section{boundary condition}

The possibility of an anechoic boundary condition transforming all the incident mechanical power into electrical power can also be investigated with the same approach. For this purpose, piezoelectric energy harvester is implemented at the boundary of a semi-infinite structure, and harvesting of the propagating bending waves are modeled using the schematic shown in Fig. 3.

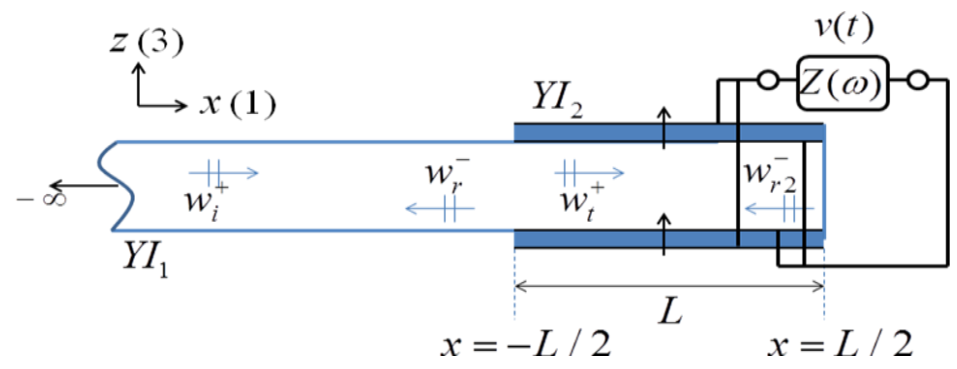

Fig. 3. Piezoelectric energy harvesting from one-dimensional bending waves using a harvester at the boundary of a semi-infinite beam. 
In this case the linear/angular displacement and force/moment equilibrium conditions at $x=-L / 2$ are still valid; however, the conditions at $x=L / 2$ are modified according to free end boundary conditions in which force and moment are equal to zero.

Imposing compatibility and equilibrium conditions at the boundaries in the mechanical domain and solving the coupled electromechanical equations, the time-averaged power flow in the beam and the time-averaged electrical power flowing to the electrical load of the harvester are calculated using Eqs. (5) and (16), respectively.

\section{Theoretical and Experimental Case Studies}

In this section three case studies are given to illustrate the application of the model and discuss its salient results. Experimental validation of the simulation studies is also performed. For all the cases, a slender aluminum beam with $25.4 \mathrm{~mm}$ width and $1.6 \mathrm{~mm}$ thickness is used $\left(\rho_{s}=2700 \mathrm{~kg} / \mathrm{m}^{3}, Y_{s}=70 \mathrm{GPa}\right)$. Note that the frequency range used in the experiments is selected such that the Euler-Bernoulli beam theory yields accurate results by satisfying the condition $\mathrm{h} / \lambda<0.1[52]$.

\subsection{Simulation results}

In the simulations continuous harmonic wave analysis is performed on the infinite and semi-infinite beams. The structure is excited by an incident propagating wave with constant amplitude. The complex amplitudes of the transmitted and reflected waves and the output voltage are obtained under different electrical loading parameters. In all case studies, the system is assumed to be ideal, i.e. without any mechanical or electrical loss. 


\subsubsection{Case study I: Energy harvesting on a uniform beam with resistive and resistive- inductive loading}

In this case, a pair of piezoelectric patches with electrodes on each side of an infinite beam is connected to a complex electrical load for energy harvesting as shown in Fig. 1(a). The piezoelectric energy harvester patches are made of PZT-5A and they are $0.267 \mathrm{~mm}$ thick and $50.8 \mathrm{~mm}$ long.

The bending wave energy harvester is first evaluated by using a resistive load connected to the piezoelectric patch terminals and the normalized output electrical power is calculated as a function of frequency for optimum resistive loads in the $0-30 \mathrm{kHz}$ range. The normalization is done by dividing the output power to the square of the incident displacement amplitude (i.e. power output per meter squared). In Fig. 4(a), normalized electrical power is plotted against the wavelength normalized length, $L \lambda$. Examining the graph along with the phase velocity calculations shows that the power output experiences a local minimum when $L$ is close to a multiple of the wavelength, as expected. Note that the behavior is not perfectly periodic in frequency due to the dispersive nature of the bending waves, i.e. the phase velocity being proportional to $\sqrt{\omega}$. The first peak, corresponding the $L \sim 3 \lambda / 4$ results in the local maximum power and power output exhibits local maxima at odd multiples of quarter wavelength, when $L \sim(2 \mathrm{n}+1) \lambda / 4, n=1,2, \ldots$

As shown in Fig. 4(a), for constant incident displacement wave amplitude, the maximum power increases with frequency under optimal resistive loading because the incident power increases. Another useful metric for comparing different load conditions is the efficiency of the energy harvester. In this case, the harvested power is normalized to the mechanical power of the incident wave (Eqn. (5)). Fig. 4(b) shows the efficiency of the harvester as a function of 
normalized length. In this case, the peaks occur when $L$ is approximately an odd multiple of $\lambda / 2$. This is expected as at higher frequencies where $L$ is larger than the wavelength, only the mechanical energy in the last $\lambda / 2$ length of the patch is efficiently converted to electrical domain, similar to the reduced coupling coefficient in higher order modes of piezoelectric resonators [53]. Also, it is seen that, with the non-dimensional representation of the patch length, the efficiency plot reduces to a unified curve for different patch lengths. Hence, once the frequency range for harvesting is determined, harvester dimensions can be chosen to work efficiently at those frequencies as long as the resistor value is also chosen optimally.

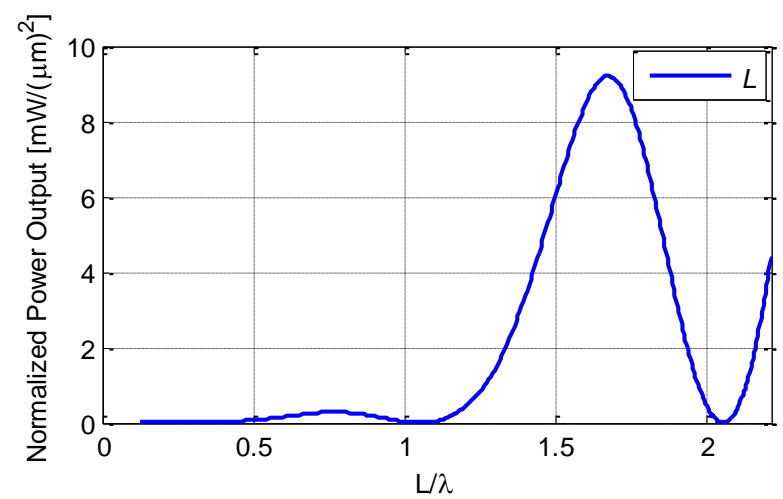

(a)

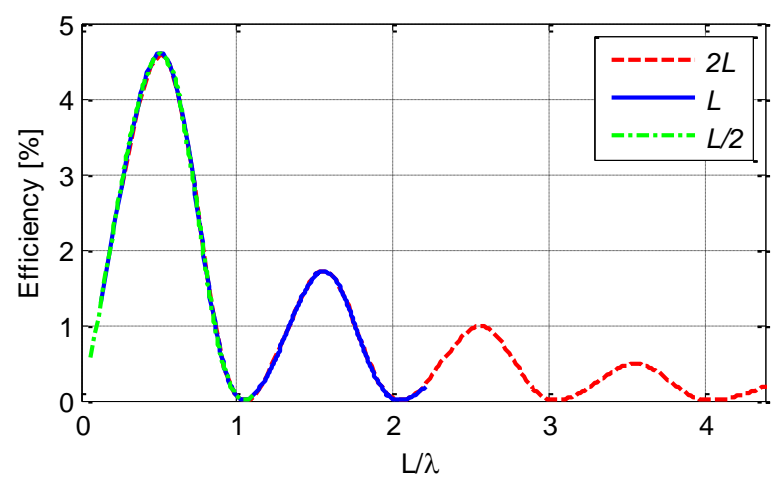

(b)

Fig. 4. Variation of (a) the harvested power and (b) the power conversion efficiency with normalized patch length under optimal resistive loading.

The optimal load resistance can be identified through 3D plots of harvested power and conversion efficiency as a function of resistance and normalized patch length. In Figs. 5(a) and (b), harvested power and power conversion efficiency are plotted, respectively, in the $0-8 \mathrm{kHz}$ range. In Fig. 5(a) the local maximum is obtained around $R_{l}=400 \Omega$ at $L \sim 3 \lambda / 4$ with minima around frequencies where $L$ is a multiple of wavelength. In Fig. 5(b) the corresponding plot for conversion efficiency is shown, where the global maximum for efficiency is $\sim 4.6 \%$ for $R_{l}=$ 
$900 \Omega$ at $L \sim \lambda / 2$. In comparing Figs. 5 (a) and (b), one realizes that the maximum power peak shifts toward higher frequencies where $L \sim \lambda$, since the input power increases with frequency. In contrast, the conversion efficiency peak is at a lower frequency, roughly corresponding to the half wavelength condition.
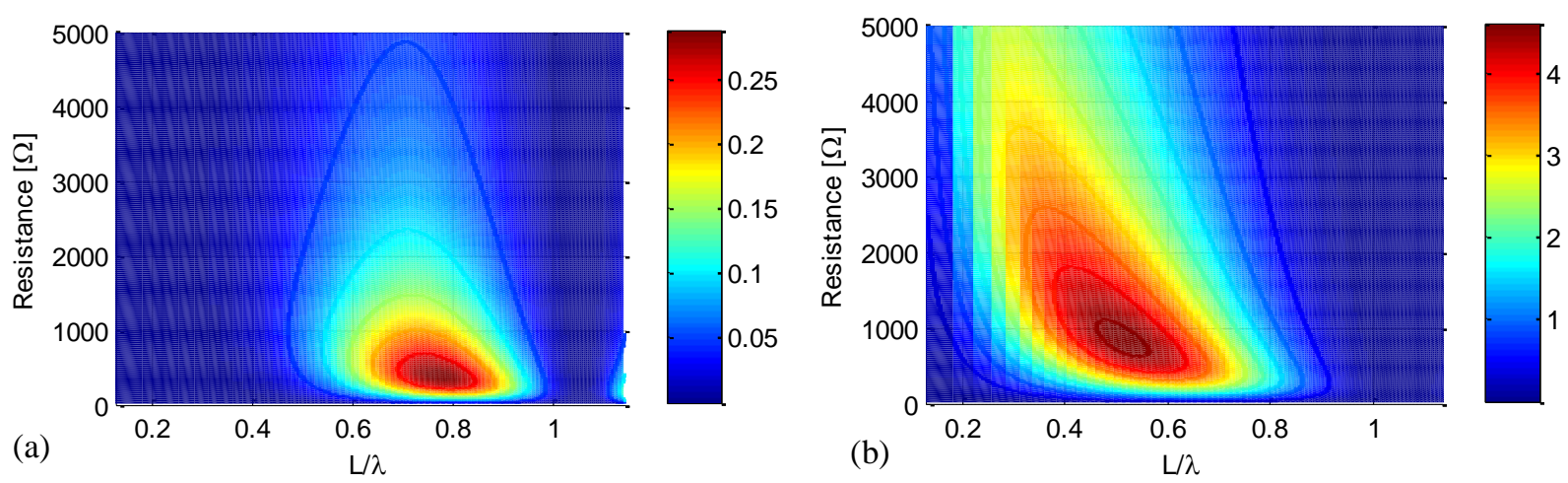

Fig. 5. Variation of (a) the harvested power $\left(\mathrm{mW} / \mu \mathrm{m}^{2}\right)$ and (b) the power conversion efficiency (\%) with load resistance and normalized patch length for resistive loading case.

These results, essentially based on wavelength matching, are obtained by others through analyses which ignored backward coupling (for example cf. Fig. 10 in [45] for $A_{0}$ mode Lamb waves, which are similar to bending waves especially at low frequencies). The impact of a full model for optimizing the efficiency of energy harvesting is more clearly seen when complex conjugate electrical impedance matching is used $[50,51,54]$. For example, in the case of a capacitive source such as the piezoelectric patch, one can achieve electrical impedance matching using an inductance in parallel with the load resistance, so that the load impedance becomes

$$
Z_{l}(\omega)=\left(\frac{1}{R_{l}}+\frac{1}{j \omega L_{l}+R_{\text {ind }}}\right)^{-1}
$$


where $L_{l}$ is the inductance and $R_{\text {ind }}$ is the internal resistance of the non-ideal inductor. Note that, in the simulations, all components are assumed to be lossless (i.e. $\eta=\tan \delta=R_{\text {ind }}=0$ ), i.e. harvester characterization is performed under ideal conditions.

Typically for maximum power transfer at a particular frequency the inductance value is chosen as $L_{l}=1 /\left(\omega^{2} C_{p}^{e q}\right)$ to eliminate the capacitive component of the impedance and the resistance value is varied as the free parameter. First set of results in Fig. 6 shows the consequence of this approach, which provides $50 \%$ efficiency over a range of normalized patch lengths, a significant improvement over the resistive case. However, by choosing both the inductance and resistance values for complex conjugate matching of the impedance at the piezoelectric patch terminals, this range can be extended except for a narrow region around $L \sim \lambda$. This approach makes the optimal condition nearly independent of the patch length, and effectively frequency, which means that high conversion efficiencies can be obtained with small piezo patches by adjusting electrical load. . The inductance values rendering the maximum efficiency at different frequencies are normalized by $1 /\left(\omega^{2} C_{p}^{e q}\right)$ as illustrated in Fig. $6(\mathrm{~b})$. This graph shows that most probably the evanescent waves, especially effective at short patch lengths and multiples of $\lambda$, contribute to the imaginary part of the electrical impedance changing the optimal tuning inductance from $1 /\left(\omega^{2} C_{p}^{e q}\right)$.

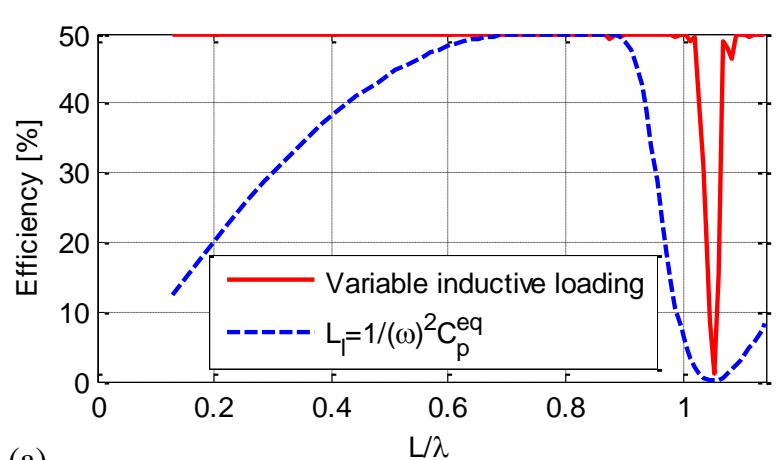

(a) (b)

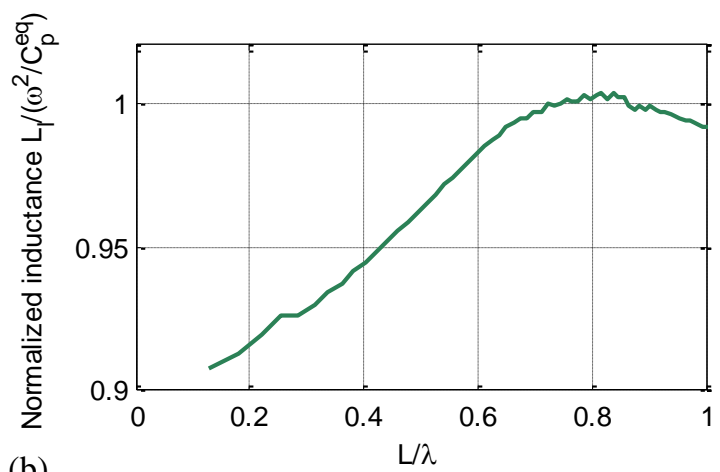

$L / \lambda$ 
Fig. 6. Variation of (a) the harvested power efficiency with normalized patch length under optimal resistive-inductive loading and (b) the corresponding normalized inductance.

Following a similar analysis to the resistive load case, in Fig. 7(a) the normalized power output is plotted as a function of resistance and non-dimensional wavelength under impedance matching conditions. It is seen that the peak frequency shifts to the higher end of the frequency band, limited by the $L \sim \lambda$ condition. Fig. 7(b) shows conversion efficiency for the same loading condition in which the maximum efficiency is limited to $50 \%$, as expected from a passive, reciprocal 3-port device with one conjugate matched port [56]. In this case, the impedance matched port is the piezoelectric patch terminals and the mechanical port terminations are symmetrical. A simple analysis of the scattering matrix for this case indicates that the rest of the mechanical power incident from the left side of the beam is equally reflected back (25\%) and transmitted $(25 \%)$ to the other side of the patch.

It is noted that unlike energy harvesting applications from vibrations of finite structures with standing waves (i.e. modal vibrations) which can be modeled with a simpler formulation and lends itself to Den Hartog's invariant point concept to obtain optimal loading parameters, in the case of wave energy harvesting the complexity of the problem prevents closed form expressions for optimization even only bending waves are considered [55]. 

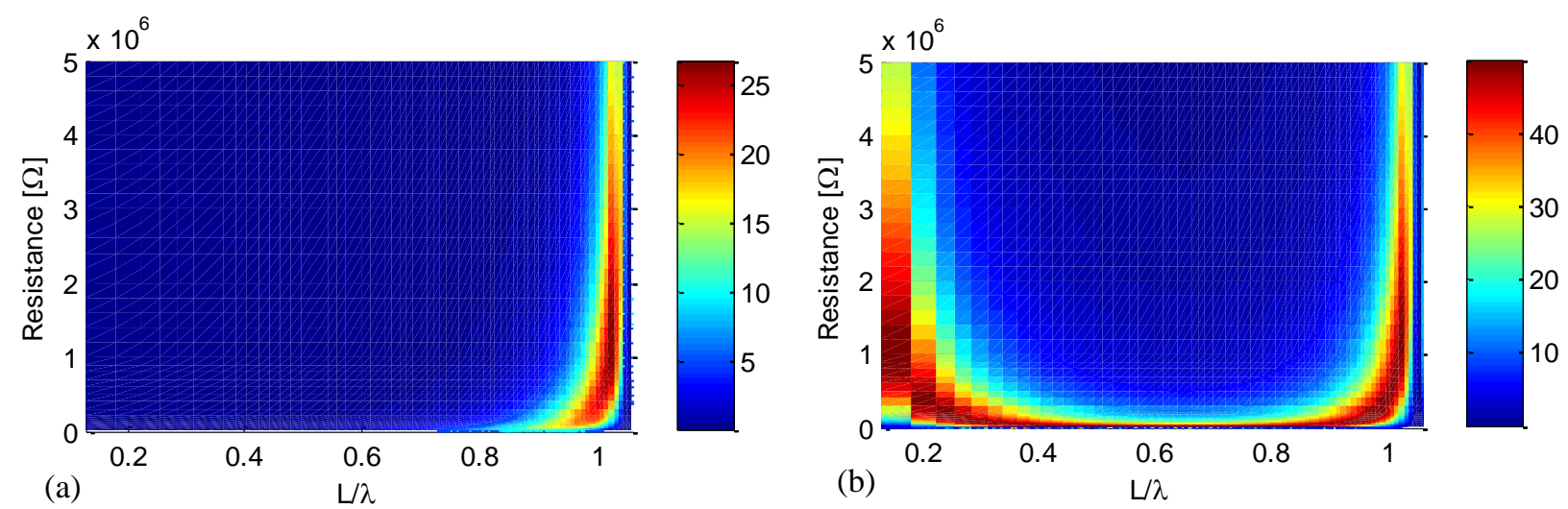

Fig. 7. Variation of (a) the harvested power $\left(\mathrm{mW} / \mu \mathrm{m}^{2}\right)$ and (b) the power conversion efficiency (\%) with load resistance and normalized patch length for parallel resistive-inductive loading case.

\subsubsection{Case study II: Energy harvesting enhancement by using a lumped obstacle}

As can be seen in Fig. 2, when a lumped obstacle is introduced, part of the bending wave energy is trapped between the harvester and the lumped obstacle resulting in a variation of the harvested power. In this case study, a pair of rectangular steel pieces (with $12.7 \mathrm{~mm}$ height, $3.175 \mathrm{~mm}$ length and $25.4 \mathrm{~mm}$ width) is considered as lumped mass (16 grams) obstacle. The same piezoelectric patches are implemented as the energy harvesting interface connected to the complex electrical load with optimal parameters obtained for maximum efficiency.

Variation of the harvester efficiency by means of a lumped mass obstacle is simulated under resistive (at $2.5 \mathrm{kHz}$ ) and resistive-inductive loading (at $1.5 \mathrm{kHz}$ ) cases with changing obstacle location in the transmitted wave domain. Fig. 8(a) and (b) show the calculated variation of the conversion efficiency under continuous wave excitation with lumped mass location normalized to wavelength $\lambda$ at the excitation frequency for resistive and resistive-inductive loading, respectively. As expected from standing wave patterns, the variation of harvester 
efficiency has regular maxima and minima with $\lambda / 2$ periodicity. The harvester efficiency without the obstacle is constant at this frequency, and this value is also included in the plots for comparison purposes. Hence, it can be concluded that the efficiency of the harvester can be increased dramatically with the addition of the symmetric lumped mass. The results also show that there is a distance limit below which the optimal conditions may not be achieved. This limit is $\sim \lambda / 4$, where evanescent fields are significant as shown in Fig. $8(\mathrm{c})$. The high efficiency values close to zero mass distance would not be practical due to limited mass dimensions.
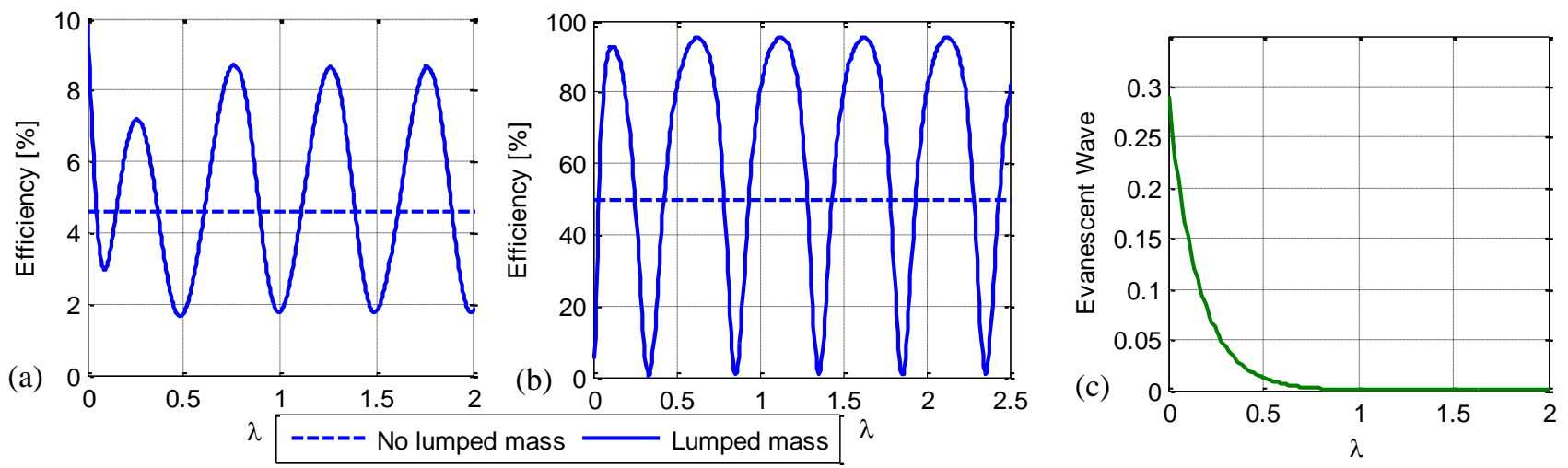

Fig. 8. Change in harvester efficiency via lumped mass under (a) resistive loading at $1.5 \mathrm{kHz}$, (b) resistive-inductive loading at $2.5 \mathrm{kHz}$; (c) normalized evanescent wave amplitude versus wavelength.

\subsubsection{Case study III: Multifunctional energy harvester - anechoic boundary condition}

In this case study, the energy harvester patch is placed at one boundary of the semiinfinite structure to effectively implement a multifunctional energy harvester which also realizes an anechoic boundary condition. The same material and dimensional properties given in the previous case studies are used except for the length of the piezoelectric energy harvester. In the simulations, 33mm long patches are used as the energy harvesting interface bonded at the end of 
the beam. Thus, high frequency harvesting is enabled in order to eliminate the limitations of the experimental setup which will be explained in Section 3.2.3.

The efficiency of the multifunctional harvester is obtained for different frequencies between $0-15 \mathrm{kHz}$ under optimal resistive-inductive loading based on conjugate impedance matching. The efficiency versus normalized patch length is shown in Fig. 9(a). Optimal loading parameters are obtained through 3D plots of conversion efficiency as a function of resistance, inductance and normalized patch length, and plotted in Fig. 9(b). It can be observed that theoretically $100 \%$ efficiency can be obtained at different frequencies by conjugate impedance matching. In practice the efficiency would decrease to some extent due to parasitic losses as will be seen in the experimental results.

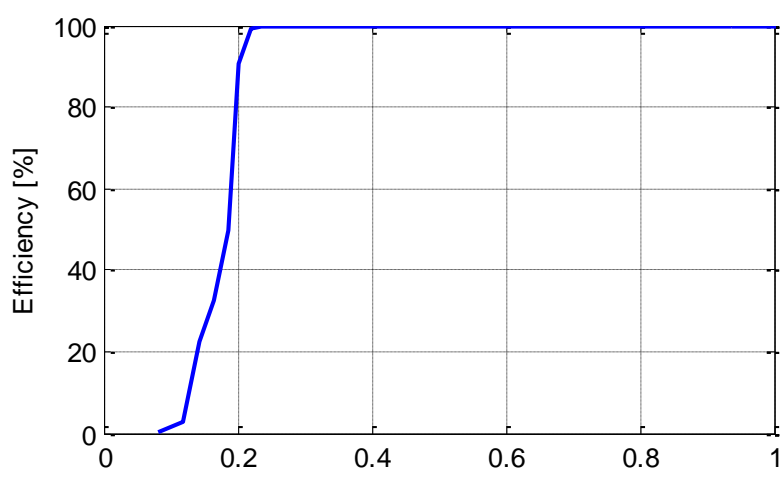

(a)

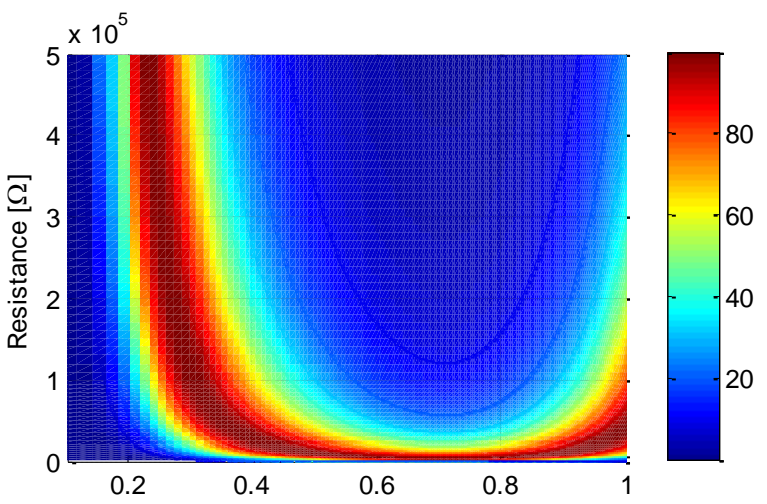

(b)

Fig. 9. Variation of (a) the harvested power efficiency with normalized patch length under optimal resistive-inductive loading and (b) the power conversion efficiency with load resistance and normalized patch length for parallel resistive-inductive loading case. 


\subsection{Experimental setup and results}

In the experiments a pair of piezoelectric patches is used as the energy harvesting interface connected to a complex electrical load (resistance and inductance). A long slender beam supported by soft foam at several locations is used as the bending wave propagation medium and Laser Doppler Vibrometers (LDVs) are used to measure the incident, transmitted, and reflected waves generated by a piezoelectric actuator as shown in Fig. 10. The LDV close to the actuator measures both the incident and reflected waves from the harvester, while the second LDV measures transmitted waves passing through the harvester.

In order to prevent spurious interference between reflected waves from the end of the beam and the propagating waves in the harvester domain, the number of cycles of the input excitation to the piezoelectric actuator is limited. Furthermore, the internal resistance of the inductance box, structural damping factor, and dielectric loss of the piezoelectric patches are included in the experimental analysis. The structural damping factor is identified for each frequency through separate experiments by taking two simultaneous LDV measurements at known distances of freely propagating waves and considering the power levels. A constant dielectric loss is extracted from the difference between the measured and calculated output voltage signals. As a result, the structural damping factor in the beam is taken as 0.0025 at 1.5 $\mathrm{kHz}$ and the dielectric loss in the piezoelectric patch is taken as $2 \%$ (accounting for the dielectric loss caused by the epoxy layers) in the calculations. 


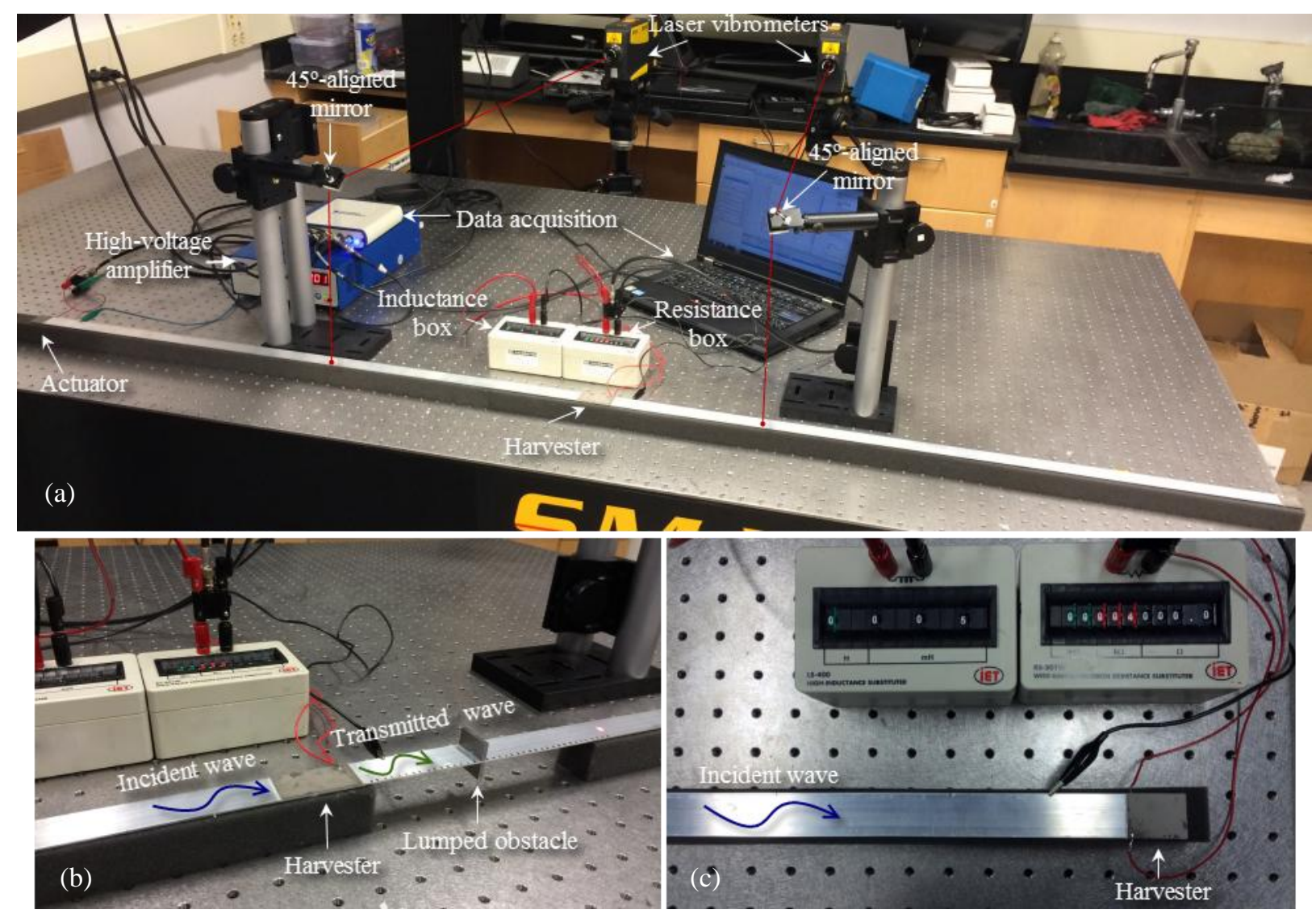

Fig. 10. Experimental setups: (a) Case study I with no lumped obstacle, (b) case study II with lumped obstacle case, and (c) case study III with energy harvester at one boundary.

\subsubsection{Case study I: Resistive and resistive-inductive loading with no obstacle}

The experimental setup shown in Fig. 10(a) is first used to test the validity of the results from Section 3.1.1. Using the LDV signal for the incident wave, the waveforms for the output voltage, transmitted and reflected velocity are calculated via transient analysis with the theoretical frequency domain transfer function and compared with the measured waveforms. For instance, the voltage output is calculated by inserting the measured incident velocity in the following formula:

$$
V(t)=\operatorname{IFFT}\left\{\operatorname{FFT}\left\{\text { Velocity }_{i n c}(t)\right\} \times G(\omega) \times e^{-j k_{b 1}(\omega) L}\right\}
$$


where $G(\omega)$ is the theoretical transfer function between the incident velocity and the voltage response, $k b_{1}$ is the theoretical wavenumber and $L$ is the distance between the harvester and LDV location.

The center frequencies of the signals used for comparison are selected to correspond to the maximum power and efficiency based on Figs. 4 and 5. For example, at $1.5 \mathrm{kHz}$ where $L \sim 0.8$ $\lambda 900 \Omega$ resistance is found and used as the optimal resistive loading. Similarly, at $2.5 \mathrm{kHz}$ where $L \sim 0.5 \lambda 8 \mathrm{k} \Omega$ resistance and $35 \mathrm{mH}$ inductance (with $13.2 \Omega$ internal resistance) is used for optimal resistive-inductive loading. Fig. 11 shows the results comparing measured and calculated results for the resistive and resistive-inductive loading case the left and right columns, respectively. The top row of Fig.11 shows the measured incident wave LDV signals. The calculated output voltage waveforms (second from the top row) and transmitted waveforms perfectly match the measurements. Note that the voltage and power levels obtained for resistive-inductive loading are significantly higher than resistive loading as expected from the simulations. The reflected velocity waveforms agree reasonably well with the measurements considering the low signal levels as compared to transmitted waves. 
(a)
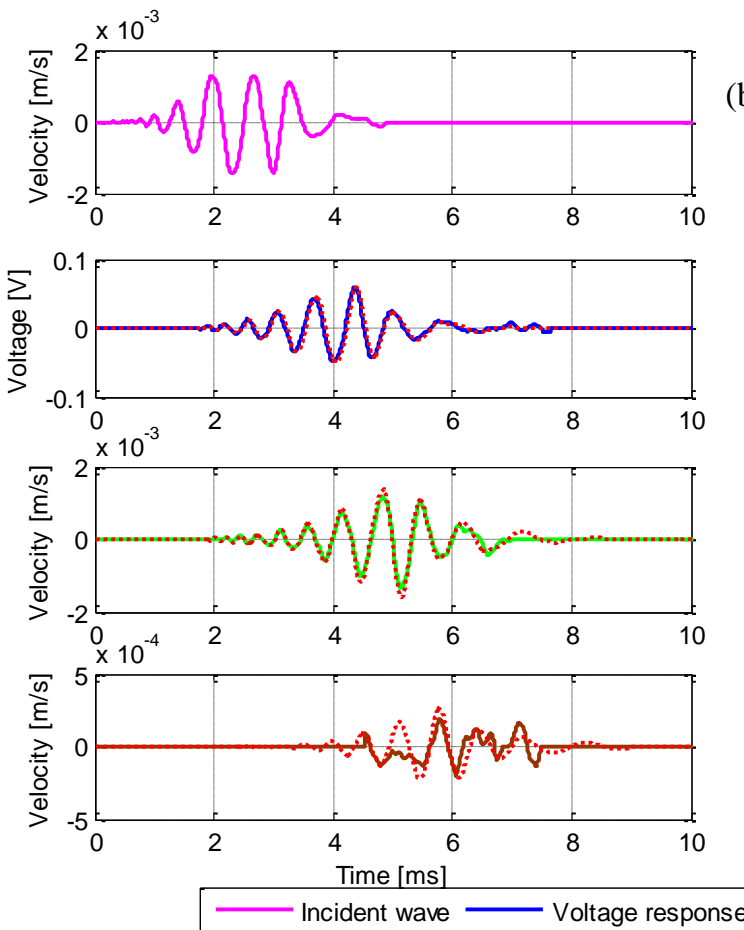

(b)
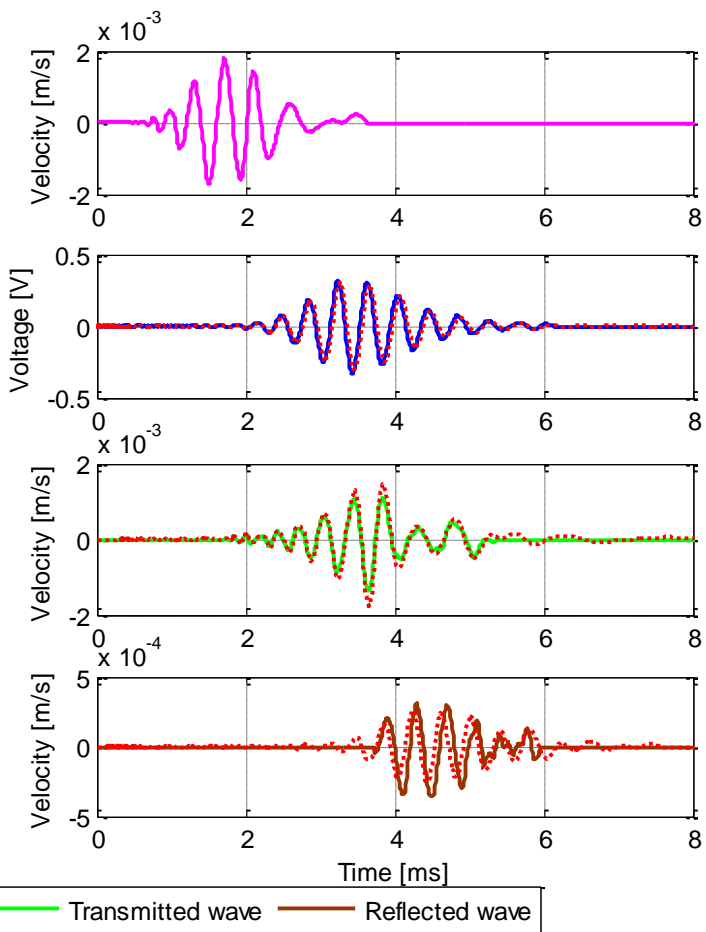

Fig. 11. Comparison of the theoretical and experimental voltage response and transmitted and reflected wave behaviors along with the measured incident waves under (a) resistive loading at $1.5 \mathrm{kHz}$ and (b) resistive-inductive loading at $2.5 \mathrm{kHz}$ (solid curves: measured; dashed curves: calculated).

For further validation, the experiments are repeated under optimal loading conditions for different frequencies between $500 \mathrm{~Hz}-8 \mathrm{kHz}$. Harvested power is obtained by dividing the power spectral density (PSD) value of the piezoelectric voltage response at the harvesting frequency by the load resistance. Similarly, average power carried in the incident wave is calculated by multiplying the PSD of the incident wave packet with $Y I_{1} k_{b 1}{ }^{3} / \omega$ resulting from Eqn. (5). Fig. 12 shows the comparisons of harvested power and efficiency under optimal resistive and resistiveinductive loading conditions. As can be seen in Fig. 12(a), the experimental maximum power is around $3 \mathrm{kHz}$ for resistive loading and $4.5 \mathrm{kHz}$ for the resistive-inductive loading, similar to 
predictions. Furthermore, experimental maximum efficiency is $4.5 \%$ at $1.5 \mathrm{kHz}$ and $26 \%$ at 2.5 $\mathrm{kHz}$ for resistive and resistive-inductive loading, respectively (Fig. 12(b)). Note that the decrease in the maximum efficiency from $31 \%$ (including the inductance loss) to $26 \%$ is because of the effect of mechanical and dielectric losses in the system. Despite of the discrepancies in the measured and calculated efficiency and power output levels (arising from the slight differences in the wave packets), the experiments at different frequencies can be considered in satisfactory agreement with the simulations. These results along with the waveform comparisons validate the proposed bending wave energy harvester model.
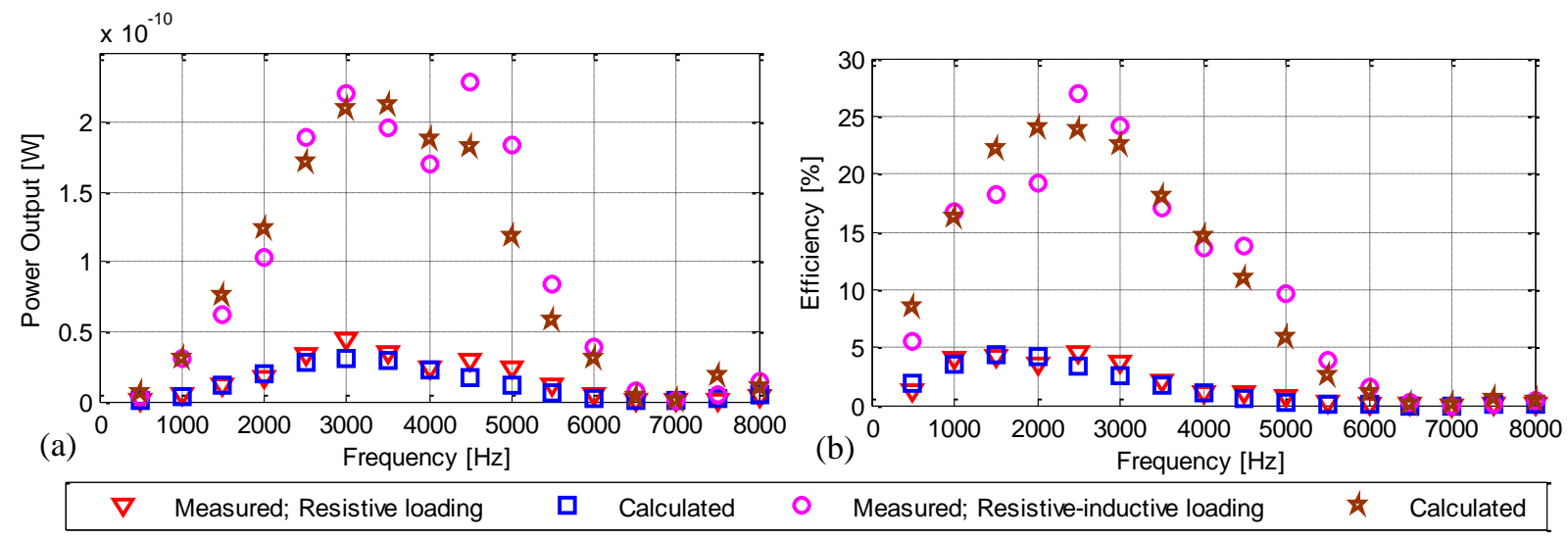

Fig. 12. Comparison of the theoretical and experimental (a) maximum power and (b) maximum efficiency with the optimal parameters of resistive and resistive-inductive loading.

\subsubsection{Case Study II: Power enhancement by using a lumped obstacle}

The setup in Fig. 10(b) with the same piezoelectric energy harvester is used to test the validity of the theoretical model for energy harvesting improvement with lumped obstacles. In Fig. 13, efficiency versus normalized lumped mass position is plotted for optimal resistive and resistive-inductive loading cases at $1.5 \mathrm{kHz}$, and $2.5 \mathrm{kHz}$, respectively. The lumped mass is 
located in $5 \mathrm{~mm}$ increments in the transmitted wave domain. Note that the appearance of maximum peaks with $\lambda / 2$ periodicity, the significant difference in efficiency with inductiveresistive loading and the asymmetric variation of the efficiency as compared to no-obstacle case all agree very well qualitatively with the predictions in Fig. 8. Transient analysis comparisons in Fig. 14 also show excellent agreement with respect to the shape of the waveforms and signal levels. The effect of evanescent waves for small normalized mass obstacle distances are also observed qualitatively as the periodic curves are distorted as the distance is reduced below $0.2 \lambda$.
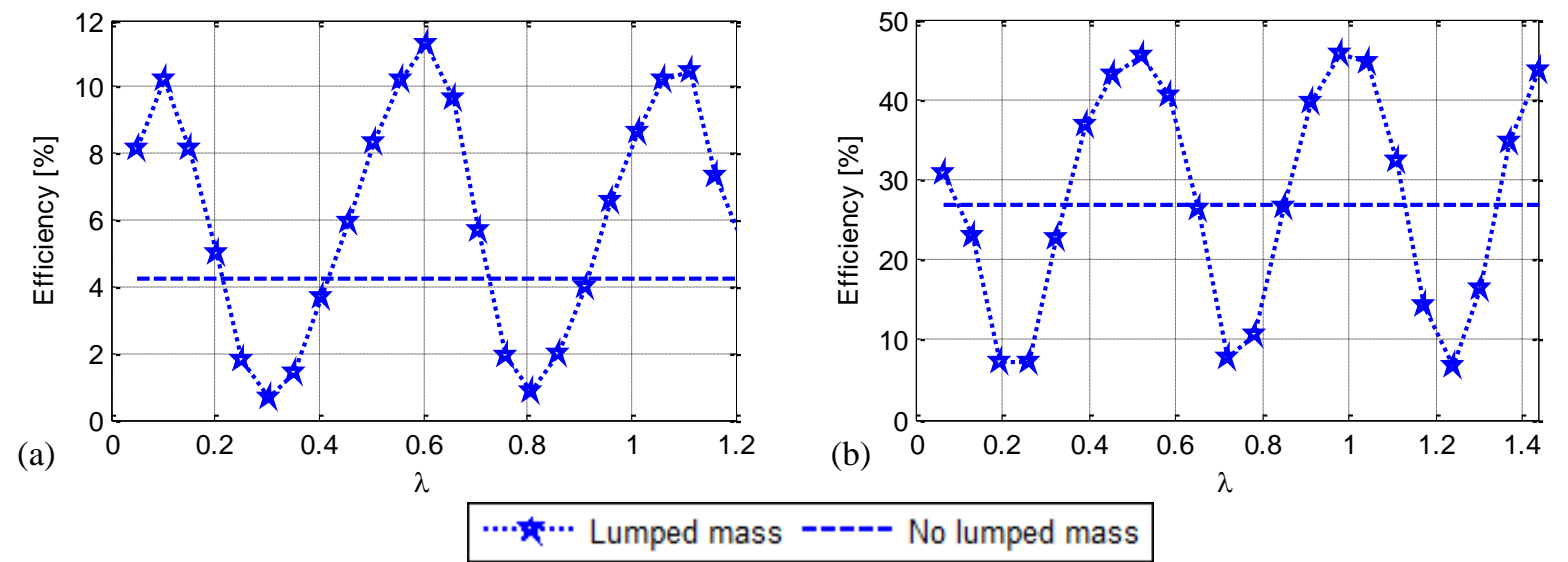

Fig. 13. Experimental power enhancement via lumped mass under, (a) resistive loading at $1.5 \mathrm{kHz}$, (b) resistive-inductive loading at $2.5 \mathrm{kHz}$.

(a)
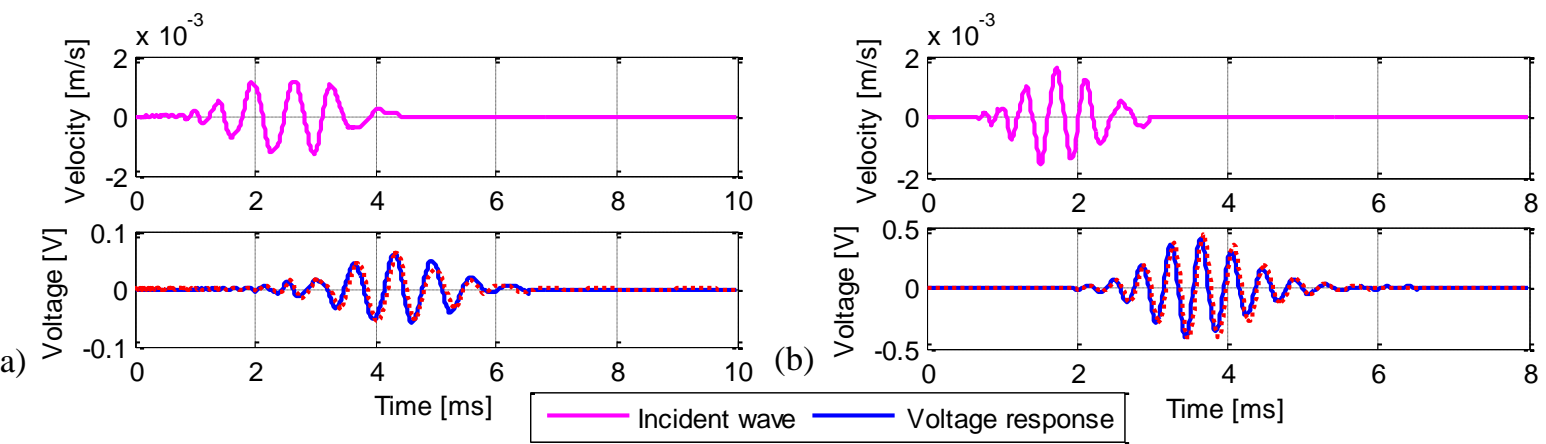
Fig. 14. Comparison of the theoretical and experimental voltage response along with the measured incident waves under (a) resistive loading at $1.5 \mathrm{kHz}$, (b) resistive-inductive loading at $2.5 \mathrm{kHz}$ (solid curves: measured; dashed curves: calculated).

\subsubsection{Case study III: Multifunctional energy-harvesting quasi-anechoic boundary condition}

The multifunctional energy harvester concept (Fig. 10(c)) analyzed in Section 3.1.3 is also experimentally tested. In contrast with the ideal lossless case leading to $100 \%$ harvester efficiency, a lower figure is expected due to losses such as the internal resistance of the inductance box, mechanical loss in the system and the dielectric losses in the harvester. Therefore, instead of a 100\% efficient anechoic boundary condition, a quasi-anechoic boundary condition performance is expected. In order to avoid the interference of the incident wave with the reflected wave, the harvester is tested at a higher frequency (at $7.95 \mathrm{kHz}$ ) with 5 cycles of burst excitation. For electrical loading, the optimal parameters for this particular frequency is used $\left(R_{l}=4 \mathrm{k} \Omega, L=5 \mathrm{mH}\right.$ and $\left.R_{\text {ind }}=3.4 \Omega\right)$, and LDV measurement at a single location is used to record the incident and reflected waves. Fig. 15(a) shows the comparison of the calculated and measured incident wave amplitude, the harvester output voltage and the reflected wave amplitude. In addition to showing excellent agreement, the notch in the reflected waveform shows that the harvester is effectively anechoic at a certain frequency contained in this wavepacket. 

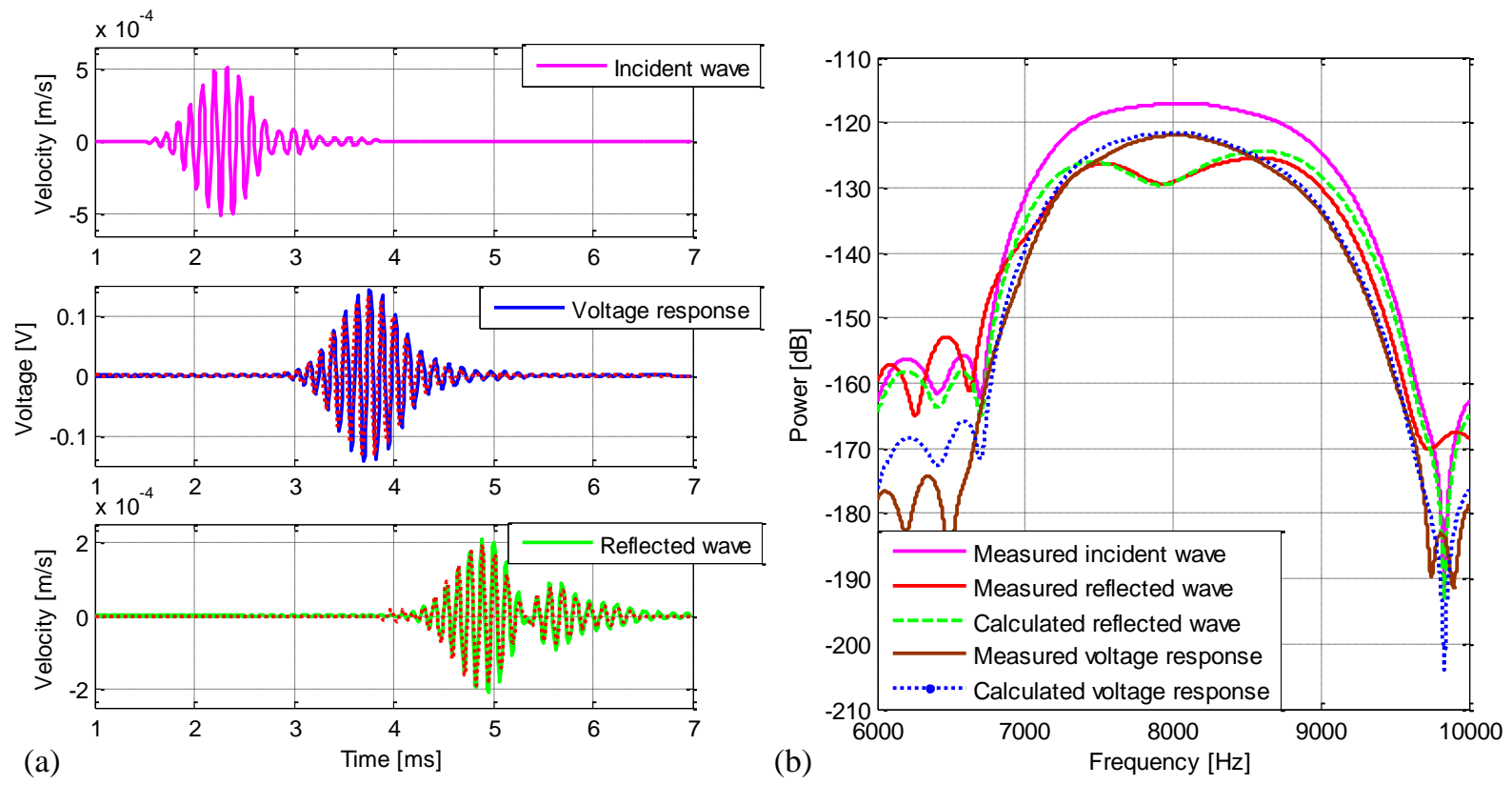

Fig. 15. Comparison of the theoretical and experimental voltage response and reflected wave behaviors along with the measured incident waves: (a) Transient analysis and (b) PSD analysis (solid curves: measured; dashed curves: calculated).

The particular anechoic frequency is much better delineated by the PSD analysis shown in Fig. 15(b). The incident wave hasa range of frequencies in the $7-9 \mathrm{kHz}$ range due to the toneburst excitation. As a result, the reflected wave includes those frequencies. However, the reflection is clearly minimized at the targeted frequency of $7.95 \mathrm{kHz}$ with optimal loading conditions. Calculations including the losses in the system predict a power reflection coefficient of $5.7 \%$ at this frequency, which agrees very well with the measured $6 \%$. In terms of harvester performance, calculations show that at this frequency, the addition of the internal loss of the inductor reduces the efficiency to $82 \%$. Inclusion of mechanical and dielectric losses in the system further reduces the efficiency to $36 \%$ which compares very well to the measured efficiency of $32 \%$. 


\section{Conclusions}

In this paper an electroelastic modeling framework is proposed for a piezoelectric energy harvester exploiting one-dimensional bending waves propagating in infinite and semi-infinite beams. The fully coupled model that accounts for piezoelectric structure and complex electrical load is based on the wave equation solution and the piezoelectric constitutive relations coupled in the equilibrium and compatibility conditions at the harvester boundaries and the electrical circuit equation. The model is used to obtain the time averaged power flow in the beam and the timeaveraged electrical power flowing to the harvester as well as the efficiency of the harvester. With the simulation case studies, wavelength matching, resistive and resistive-inductive circuits, and performance enhancement by a localized obstacle are explored. A substantial performance enhancement is observed in ideal conditions by resistive-inductive loading as compared to the resistive loading case with more than an order of magnitude increase in the maximum power output and $4.5 \%$ to $50 \%$ improvement in the maximum efficiency over a larger frequency range. In the case of power enhancement method with spatially localization of a lumped obstacle, the harvested power is almost doubled by properly locating the obstacle with respect to the harvester and the maximum efficiency of $95 \%$ is obtained under resistive-inductive loading. Additionally, a multifunctional piezoelectric energy harvester - anechoic boundary condition is proposed, in which nearly all the energy in a propagating bending wave can be extracted using piezoelectric patches at the free end of a beam. The harvester is tested under these different configurations, and the proposed work is validated with the experiments. Through transient and power analyses, the experimental and theoretical results are compared and an excellent agreement is observed. Due to the losses in the system, harvester efficiency decreases to $26 \%$ for resistive-inductive loading and $45 \%$ efficiency is obtained with the lumped mass addition. On the other hand, in the 
anechoic boundary condition experiments, a $6 \%$ power reflection ratio is obtained, resulting in quasi-anechoic behavior, which is very promising for achieving an energy-harvesting boundary condition that minimizes the reflections. These enhancement methods are most effective and practical when piezoelectric patch lengths and obstacle to patch distances are $\sim>\lambda / 4$, where evanescent fields become insignificant, while the model can readily accommodate the presence of evanescent waves for arbitrary patch lengths. While the present effort explored electroelastic dynamics of bending waves in an infinite/semi-infinite beam shunted to an AC circuit (with linear resistive and reactive components for linear impedance matching), future work may investigate nonlinear energy harvesting circuits with standard AC-DC conversion [57, 58], switching circuits $[59,60]$ for boosted power output in case of weak electromechanical coupling, as well as nonlinear impedance matching circuits [51].

\section{Acknowledgment}

This work was supported in part by the National Science Foundation under Grant No. CMMI-1333978.

\section{Appendix A}

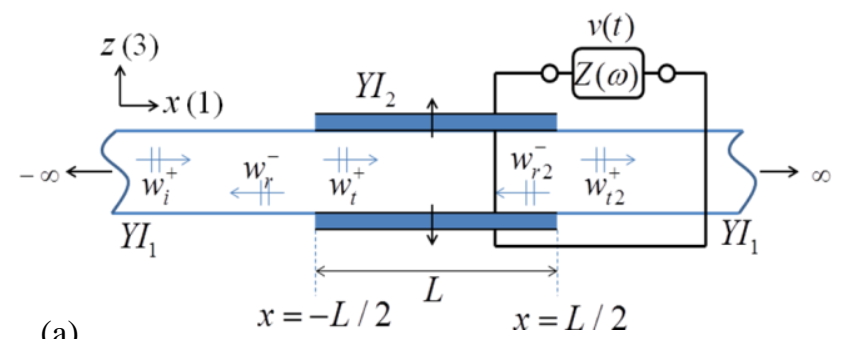

(a)

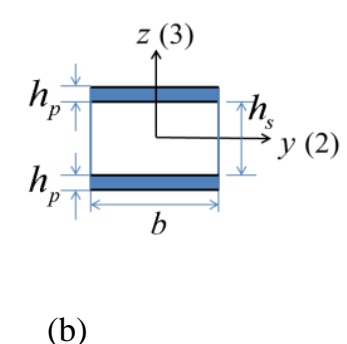

(b)

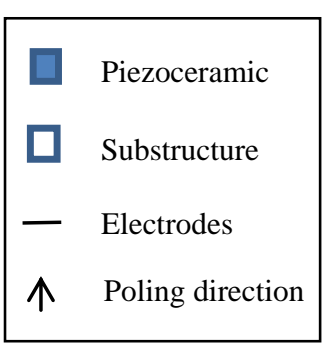

个 Poling direction

Fig. A.16. Incident, reflected, and transmitted waves at discontinuities: (a) Series connection and (b) composite cross-section in the harvester region. 
In the series connection case, the voltage across the electrodes of each piezoceramic layer is $v(t) / 2$ [3]. And due to opposite poling in the series connection, the instantaneous electric fields are in the same direction (i.e., $E_{3}(t)=-v(t) / 2 h_{p}$ for both layers). Substituting Eqs. (7) into Eq. (6), the backward coupling term in Eqn. (8) is obtained.

$$
\chi=\frac{b \bar{e}_{31}}{2 h_{p}}\left[\left(h_{p}+\frac{h_{s}}{2}\right)^{2}-\left(\frac{h_{s}}{2}\right)^{2}\right]=b \bar{e}_{31} h_{p c}
$$

The equivalent capacitance of two identical capacitors $\left(\bar{\varepsilon}_{33}^{S} b L / h_{p}\right)$ connected in series is

$$
C_{p}^{e q}=\bar{\varepsilon}_{33}^{S} b L / 2 h_{p}
$$

Then, it is substituted into the electrical circuit equation given in Eqn. (12). The expression of the dependent current source for the series connection is

$$
i_{p}(t)=\tilde{I}_{p} e^{j \omega t}=-\bar{e}_{31} h_{p c} b \int_{-L / 2}^{L / 2} \frac{\partial^{3} w_{2}(x, t)}{\partial x^{2} \partial t} d x
$$

and the amplitude of the current in Eq. (A.3) can be obtained as $\tilde{I}_{p}=-j \omega \kappa$ with $\kappa$ being the forward coupling term, which is

$$
\kappa=2 k_{b 2} \bar{e}_{31} h_{p c} b\left(-\left(\tilde{C}_{1}+\tilde{D}_{1}\right) \sin \left(k_{b 2} \frac{L}{2}\right)+\left(\tilde{C}_{2}+\tilde{D}_{2}\right) \sinh \left(k_{b 2} \frac{L}{2}\right)\right)
$$

Note that, series connection is preferred for large voltage output, whereas parallel connection is preferred for large current output [3]. Both series and parallel connection configurations yield the same power output levels under optimal loading conditions which differ due to different equivalent capacitances of the two configurations. In this study, the focus is on the harvested power and the piezoelectric patches are connected in parallel in the experiments. However, we provide both configurations for the completeness of the study. 


\section{References}

[1] S. Roundy, P. K. Wright, and J. M. Rabaey, Energy scavenging for wireless sensor networks with special focus on vibrations. New York: Springer, 2004.

[2] S. Priya and D. J. Inman, Energy harvesting technologies. New York: Springer, 2009.

[3] A. Erturk and D. J. Inman, Piezoelectric energy harvesting: Wiley, 2011.

[4] N. Elvin and A. Erturk, Advances in energy harvesting methods: Springer, 2013.

[5] S. R. Anton and H. A. Sodano, "A review of power harvesting using piezoelectric materials (2003-2006)," Smart Mater. Struct. , vol. 16, 2007.

[6] S. C. Stanton, C. C. McGehee, and B. P. Mann, "Reversible hysteresis for broadband magnetopiezoelastic energy harvesting," Applied Physics Letters, vol. 95, p. 174103, 2009.

[7] R. Ramlan, M. Brennan, B. Mace, and I. Kovacic, "Potential benefits of a non-linear stiffness in an energy harvesting device," Nonlinear Dynamics, vol. 59, pp. 545-558, 2010.

[8] G. Litak, M. Friswell, and S. Adhikari, "Magnetopiezoelastic energy harvesting driven by random excitations," Applied Physics Letters, vol. 96, p. 214103, 2010.

[9] A. Erturk and D. Inman, "Broadband piezoelectric power generation on high-energy orbits of the bistable Duffing oscillator with electromechanical coupling," Journal of Sound and Vibration, vol. 330, pp. 2339-2353, 2011.

[10] R. Ramlan, M. J. Brennan, B. R. Mace, and S. G. Burrow, "On the performance of a dual-mode non-linear vibration energy harvesting device," Journal of Intelligent Material Systems and Structures, p. 1045389X12443017, 2012.

[11] K. A. Cook-Chennault, N. Thambi, and A. M. Sastry, "Powering MEMS portable devices-a review of non-regenerative and regenerative power supply systems with special emphasis on piezoelectric energy harvesting systems," Smart Mater. Struct. , vol. 17, 2008.

[12] A. Erturk and D. J. Inman, "An experimentally validated bimorph cantilever model for piezoelectric energy harvesting from base excitations," Smart Mater. Struct., vol. 18, 2009.

[13] A. Bibo and M. Daqaq, "Energy harvesting under combined aerodynamic and base excitations," J. Vib. Acoust. , vol. 332, pp. 5086-5102, 2011.

[14] M. Bryant and I. Garcia, "Modeling and Testing of a Novel Aeroelastic Flutter Energy Harvester," J. Vib. Acoust., vol. 133, 2011.

[15] O. Doare and S. Michelin, "Piezoelectric coupling in energy-harvesting fluttering flexible plates: linear stability analysis and conversion efficiency," J. Fluids Struct., vol. 27, pp. 1357-1375, 2011.

[16] J. A. Dunnmon, S. C. Stanton, B. P. Mann, and E. H. Dowell, "Power extraction from aeroelastic limit cycle oscillations," J. Fluids Struct., vol. 27, pp. 1182-1198, 2011.

[17] A. Erturk, W. G. R. Vieira, C. De Marqui, and D. J. Inman, "On the energy harvesting potential of piezoaeroelastic systems," App. Phys. L., vol. 96, 2010.

[18] C. K. Kwuimy, G. Litak, M. Borowiec, and C. Nataraj, "Performance of a piezoelectric energy harvester driven by air flow," Applied Physics Letters, vol. 100, p. 024103, 2012.

[19] C. De Marqui, W. G. Vieira, A. Erturk, and D. J. Inman, "Modeling and analysis of piezoelectric energy harvesting from aeroelastic vibrations using the doublet-lattice method," Journal of Vibration and Acoustics, vol. 133, p. 011003, 2011. 
[20] H. D. Akaydin, N. Elvin, and Y. Andreopoulus, "Wake of a cylinder: a paradigm for energy harvesting with piezoelectric materials," Exp. Fluids vol. 49, pp. 291-304, 2010.

[21] J. J. Allen and J. Smits, "Energy harvesting eel," J. Fluids Struct., vol. 15, 2000.

[22] S. Pobering, S. Ebermeyer, and N. Schwesinger, "Generation of electrical energy using short piezoelectric cantilevers in flowing media," in Proc. SPIE 7288, 2009.

[23] A. Erturk and G. Delporte, "Underwater thrust and power generation using flexible piezoelectric composites: an experimental investigation toward self-powered swimmersensor platforms," Smart materials and Structures, vol. 20, p. 125013, 2011.

[24] C. J. Rupp, M. L. Dunn, and K. Maute, "Switchable phononic wave filtering, guiding, harvesting, and actuating in polarization-patterned piezoelectric solids," App. Phys. L., vol. 96, 2010.

[25] B. Li, A. J. Laviage, J. H. You, and Y. Kim, "Harvesting low-frequency acoustic energy using multiple PVDF beam arrays in quarter-wavelength acoustic resonator," Applied Acoust., vol. 74, pp. 1271-1278, 2013.

[26] S. B. Horowitz, M. Sheplak, L. N. Cattafesta III, and T. Nishida, "A MEMS acoustic energy harvester," J. Micromechanics and Microengineering, vol. 16, 2006.

[27] F. Liu, A. Phipps, S. Horowitz, K. Ngo, L. Cattafesta, T. Nishida, et al., "Acoustic energy harvesting using an electromechanical Helmholtz resonator," J. Acoust. Soc. Am., 2008.

[28] Z. S. Chen, Y. M. Yang, Z. M. Lu, and Y. T. Luo, "Broadband characteristics of vibration energy harvesting using one dimension al phononic piezoelectric cantilever beams," Phys. B: Condens. Matter vol. 410, pp. 5-12, 2013.

[29] S. Gonella, A. C. To, and W. K. Liu, "Interplay between phononic bandgaps and piezoelectric microstructures for energu harvesting," J. Mech. Phys. Solids, vol. 57, pp. 621-633, 2009.

[30] W. C. Wang, L. Y. Wu, L. W. Chen, and C. M. Liu, "Acoustic energy harvesting by piezoelectric curved beams in the cavity of a sonic crystal," Smart Mater. Struct., vol. 19, 2010.

[31] L. Y. Wu, L. W. Chen, and C. M. Liu, "Acoustic energy harvesting using resonant cavity of a sonic crystal," App. Phys. L., vol. 95, 2009.

[32] A. Yang, P. Li, Y. Wen, C. Lu, X. Peng, J. Zhang, et al., "Enhanced acoustic energy harvesting using coupled resonance structure of sonic crystal and Helmholtz resonator," App. Phys. L., vol. 12, 2013.

[33] M. Carrara, M. R. Cacan, J. Toussaint, M. J. Leamy, M. Ruzzene, and A. Erturk, "Metamaterial-inspired structures and concepts for elastoacoustic wave energy harvesting," Smart Mater. Struct., vol. 22, 2013.

[34] M. Carrara, M. Cacan, M. Leamy, M. Ruzzene, and A. Erturk, "Dramatic enhancement of structure-borne wave energy harvesting using an elliptical acoustic mirror," Applied Physics Letters, vol. 100, p. 204105, 2012.

[35] Z. Chen, B. Guo, Y. Yang, and C. Cheng, "Metamaterials-based enhanced energy harvesting: A review," Physica B, vol. 438, pp. 1-8 2014.

[36] A. Raghavan and C. E. S. Cesnik, "Finite-dimensional piezoelectric transducer modeling for guided wave based structural health monitoring " Smart Mater. Struct., vol. 14, 2005.

[37] W. K. Chiu, S. C. Galea, and N. Rajic, "Damage detection in bonded repairs using piezoceramics " Smart Mater. Struct., vol. 9, 2000.

[38] V. Giurgiutiu, Structural health monitoring with piezoelectric wafer active sensors. New York: Elsevier Academic Press, 2007. 
[39] G. Park, C. R. Farrar, F. L. di Scalea, and S. Coccia, "Performance assessment and validation of piezoelectric active-sensors in structural health monitoring," Smart Mater. Struct., vol. 15, 2006.

[40] C. Linag, F. Sun, and C. A. Rogers, "Electro-mechanical impedance modeling of active material systems " Smart Mater. Struct., vol. 5, 1996.

[41] V. Giurgiutiu and C. A. Rogers, "Recent Advancements in the Electro-Mechanical (E/M) Impedance Method for Structural Health Monitoring and NDE," in Proc. SPIE San Diego, 1998, pp. 536-547.

[42] G. Park, H. Sohn, C. R. Farrar, and D. J. Inman, "Overview of Piezoelectric Impedancebased Health Monitoring and Path Forward," Shock and Vibration Digest, vol. 35, pp. 451-463, 2003.

[43] H. Song, H. J. Lim, and H. Sohn, "Electromechanical impedance measurement from large structures using a dual piezoelectric transducer," J Sound Vib, vol. 332, pp. 6580-6595, 2013.

[44] V. Giurgiutiu and A. Zagrai, "Characterization of piezoelectric wafer active sensors," $J$. Intell. Mater. Syst. Struct. , vol. 11, pp. 959-976, 2000.

[45] V. Giurgiutiu, "Tuned Lamb wave excitation and detection with piezoelectric wafer active sensors for structural health monitoring," J. Intell. Mater. Syst. Struct., vol. 16, 2005.

[46] B. Mace, "Wave reflection and transmission in beams," Journal of Sound and Vibration, vol. 97, pp. 237-246, 1984.

[47] F. Fahy and P. Gardonio, Sound and structural vibration-radiation, transmission and response: Elsevier, 2007.

[48] D. W. Miller and A. V. Flotow, "A travelling wave approach to power flow in structural networks," J. Sound Vib. , vol. 128, pp. 145-162 1989.

[49] B. R. Mace, "Power flow between two continuous one-dimensional subsystems: A wave solution " J. Sound Vib., pp. 289-319, 1992.

[50] J. M. Renno, M. F. Daqaq, and D. J. Inman, "On the optimal energy harvesting from a vibration source," Journal of Sound and Vibration, vol. 320, pp. 386-405, 2009.

[51] N. Kong, D. S. Ha, A. Erturk, and D. J. Inman, "Resistive impedance matching circuit for piezoelectric energy harvesting," Journal of Intelligent Material Systems and Structures, 2010.

[52] K. F. Graff, Wave Motion in Elastic Solids. New York: Dover Publications, 1991.

[53] G. S. Kino, Acoustic Waves: Devices, Imaging, and Analog Signal Processing. Englewood Cliffs, NJ: Prentice Hall, 1987.

[54] S. Jiang, Li, X., Guo, S., Hu, Y., Yang, J., Jiang, Q., "Performance of a piezoelectric bimorph for scavenging vibration energy," Smart Materials and Structures, vol. 14, 2005.

[55] N. W. Hogood and A. Von Flotow, "Damping of structural vibrations with piezoelectric materials and passive electrical networks," J. Sound Vib. , pp. 243-268, 1991.

[56] D. M. Pozar, Microwave Engineering, 4th ed.: John Wiley \& Sons, Inc, 2012.

[57] Y. Shu and I. Lien, "Analysis of power output for piezoelectric energy harvesting systems," Smart materials and structures, vol. 15, p. 1499, 2006.

[58] C. J. Rupp, M. L. Dunn, and K. Maute, "Analysis of piezoelectric energy harvesting systems with non-linear circuits using the harmonic balance method," Journal of Intelligent Material Systems and Structures, vol. 21, pp. 1383-1396, 2010. 
[59] D. Guyomar, A. Badel, E. Lefeuvre, and C. Richard, "Toward energy harvesting using active materials and conversion improvement by nonlinear processing," Ultrasonics, Ferroelectrics, and Frequency Control, IEEE Transactions on, vol. 52, pp. 584-595, 2005.

[60] Y. Shu, I. Lien, and W. Wu, "An improved analysis of the SSHI interface in piezoelectric energy harvesting," Smart Materials and Structures, vol. 16, p. 2253, 2007. 